Check for updates

Cite this: RSC Adv., 2017, 7, 40905

Received 21st June 2017

Accepted 13th August 2017

DOI: $10.1039 / c 7 r a 06852 b$

rsc.li/rsc-advances

\title{
Occurrence and attenuation of pharmaceuticals and their transformation products in rivers impacted by sewage treatment plants $\uparrow$
}

\begin{abstract}
Daoping Zha, ${ }^{a}$ Ying Li, (D) *a Li Wang, ${ }^{b}$ Cunman Yang ${ }^{a}$ and Guanghua Lu ${ }^{a}$
Pharmaceuticals and their transformation products (TPs) have been generally detected in the aquatic environment. They threaten human health and increase environmental risk, but knowledge on their fate and attenuation in environmental ecosystems is still lacking. In this study, 13 parent pharmaceuticals and 8 of their TPs in wastewater containing rivers were investigated. We detected all of the compounds except carboxyibuprofen and sulfacetamide in at least one site in all sampling points and found that sewage treatment plants were the principal source of these substances. The majority of parent pharmaceuticals were attenuated in Yangtze River and Qinhuai River, but only a small number were attenuated in the Yunliang River. All detected TPs except 4-chlorobenzoic acid were attenuated in the three rivers. Photodegradation, sorption to sediments, and biotransformation may be important attenuation processes for these substances. We found the largest extent of attenuation in the Yangtze River, where conditions for attenuation were most favorable. Extents of attenuation values for some substances obtained by active and passive sampling were roughly the same; thus, using a polar organic chemical integrative sampler may be an appropriate method for determining the extent of attenuation of organic micropollutants in rivers.
\end{abstract}

\section{Introduction}

With the increasing severity of aquatic system pollution, pharmaceuticals have become recognized as emerging contaminants. These compounds have prompted attention around the world because of their unpredictable ability to disrupt ecosystems and human health. ${ }^{1-3}$ Even at very low levels, they can have effects on the environment. ${ }^{4,5}$ They are released into rivers and streams via wastewater from sewage treatment plants (STPs), agricultural activity, and animal husbandry. ${ }^{4,6}$ The inappropriate treatment of pharmaceuticals leads to their continuous input into aquatic systems. As a result, a multitude of pharmaceuticals at nanogram-per-liter to microgram-per-liter levels have been detected in surface water, groundwater, and even in drinking water ${ }^{7-10}$ Moreover, the transformation products (TPs) of pharmaceuticals by microorganisms under natural conditions are present in aquatic environments. In aquatic environments, some TPs may form at higher concentrations or may be more toxic compared with their parent compounds. ${ }^{11-13}$ Studies

${ }^{a}$ Key Laboratory of Integrated Regulation and Resource Development on Shallow Lakes, Ministry of Education, College of Environment, HoHai University, Nanjing, Jiangsu Province 210098, China. E-mail: hj6688@hhu.edu.cn; Tel: +86-25-83786696

${ }^{b}$ Jiangsu Province Hydrology and Water Resources Investigation Bureau, Nanjing, Jiangsu Province 210029, China

$\dagger$ Electronic supplementary information (ESI) available. See DOI: $10.1039 / \mathrm{c} 7 \mathrm{ra} 06852 \mathrm{~b}$ have shown that pharmaceuticals and some of their TPs can be toxic to organisms, posing a threat to ecosystems and human health through food-chain transmission and enrichment. ${ }^{12,14}$ After entry of these pharmaceuticals and their TPs into the aquatic environment, they flow downstream with the water and eventually undergo absorption and degradation, resulting in their attenuation.

Knowledge about the attenuation of pharmaceuticals and other polar micropollutants in rivers and streams has increased, ${ }^{15-17}$ but information on the attenuation of their TPs remains limited, despite the growing number of TPs that have been detected. ${ }^{18,19}$ The majority of research into the attenuation of pharmaceuticals has been done under laboratory conditions because of the complexity of actual environmental conditions. Since application of simulation results under laboratory conditions to actual environments is limited, further research is needed to prove the feasibility of the experimental results. The attenuation of pharmaceuticals and their TPs in rivers is affected by numerous factors. Processes related to such attenuation may depend in part on water quality parameters of the rivers, such as water velocity, dissolved oxygen levels, total suspended solid concentration, temperature, and $\mathrm{pH}^{.16,20}$ Furthermore, studies have shown that cation exchange capacity, complexation, and hydrogen bonding can affect attenuation. ${ }^{21}$ These substances undergo physical (dilution and volatilization), chemical (adsorption and photolysis), and biological processes (uptake and accumulation) as they are transported downstream, 
leading to changes in their concentrations. ${ }^{20,22}$ Many approaches to calculating the attenuation of these substances have been developed. These include relative attenuation (RA), ${ }^{13,15}$ in situ attenuation ${ }^{16,22}$ and comparable model-based approach. ${ }^{20}$ The attenuation was evaluated by using first-order kinetic for latter two approaches, but tracer study was essential in order to determine travel time. While relative attenuation was calculated by using concentration of compounds between two sites. Such calculation is still significant in the assessment of attenuation of pharmaceuticals and their TPs in aquatic environments.

In studies on the fate of polar organic micropollutants in aquatic environments, samples were typically collected using active sampling. It is cost and labor intensive to collect and analyze large numbers of samples. Polar organic chemical integrative samplers (POCIS) as a complementary approach can obtain time-weighted average (TWA) concentration of these contaminants in the aquatic environment during a specific period. In addition, POCIS do not require a power supply in the field and are flexible enough to be deployed at sites that are difficult to access. POCIS have been successfully used for monitoring a various of polar organic pollutants, such as polar pharmaceuticals, endocrine disrupting compounds and pesticides. $^{23-25}$

In this study, we assessed the occurrence and attenuation of 13 pharmaceuticals and 8 TPs in rivers downstream of 3 STPs. We observed the variability of concentration and attenuation of the different pharmaceuticals. Because of the convenience and economy of using POCIS, our main objective was to explore the feasibility of using a POCIS in studying the attenuation of these substances. Simultaneously, we compared the attenuation rates of the pharmaceuticals and their TPs in the three rivers, and we analyzed the effects of different river characteristics on attenuation. Our study provides a theoretical basis for determining the attenuation of these substances in rivers.

\section{Material and methods}

\section{Reagents and materials}

Details of all reagents and materials are provided in the ESI. $\dagger$ The physical and chemical properties of all target compounds are shown in Table 1.

The POCIS consists of $200 \mathrm{mg}$ of Oasis HLB sorbent sandwiched between polyether sulfone (PES) membranes $(0.1 \mu \mathrm{m}$ pore size, $47 \mathrm{~mm}$ diameter, Lu Na Sheng Bio Technology Company, China) locked with two Plexiglas flanges. The filler was washed with methanol and ultrapure water before use. All parts of the POCIS were soaked in methanol, rinsed with ultrapure water, and then finally packed with aluminum foil.

\section{Study sites and river characteristics}

Field experiments were performed at four rivers affected by STP effluents in Nanjing, China. The four rivers were Jinchuan (JC) River and Yangtze (YZ) River, which are affected by northern city (NC) STP; Qinhuai (QH) River, which is impacted by science park STP; and Yunliang (YL) River, which is affected by eastern city STP. For the JC River and YZ River, water was sampled at five different locations: the STP effluent itself (N2); $200 \mathrm{~m}$ upstream of the STP outfall (N1); $500 \mathrm{~m}$ downstream of the STP outlet (N3), which converge with the YZ River; and at two locations at distances of $2000 \mathrm{~m}$ (N4) and $4100 \mathrm{~m}$ (N5) from N3. Therefore, the attenuation in the JC River at short distances was not computed. Five sampling points in the QH River were selected:

Table 1 Physical and chemical properties of parent substances and transformation products (TPs)

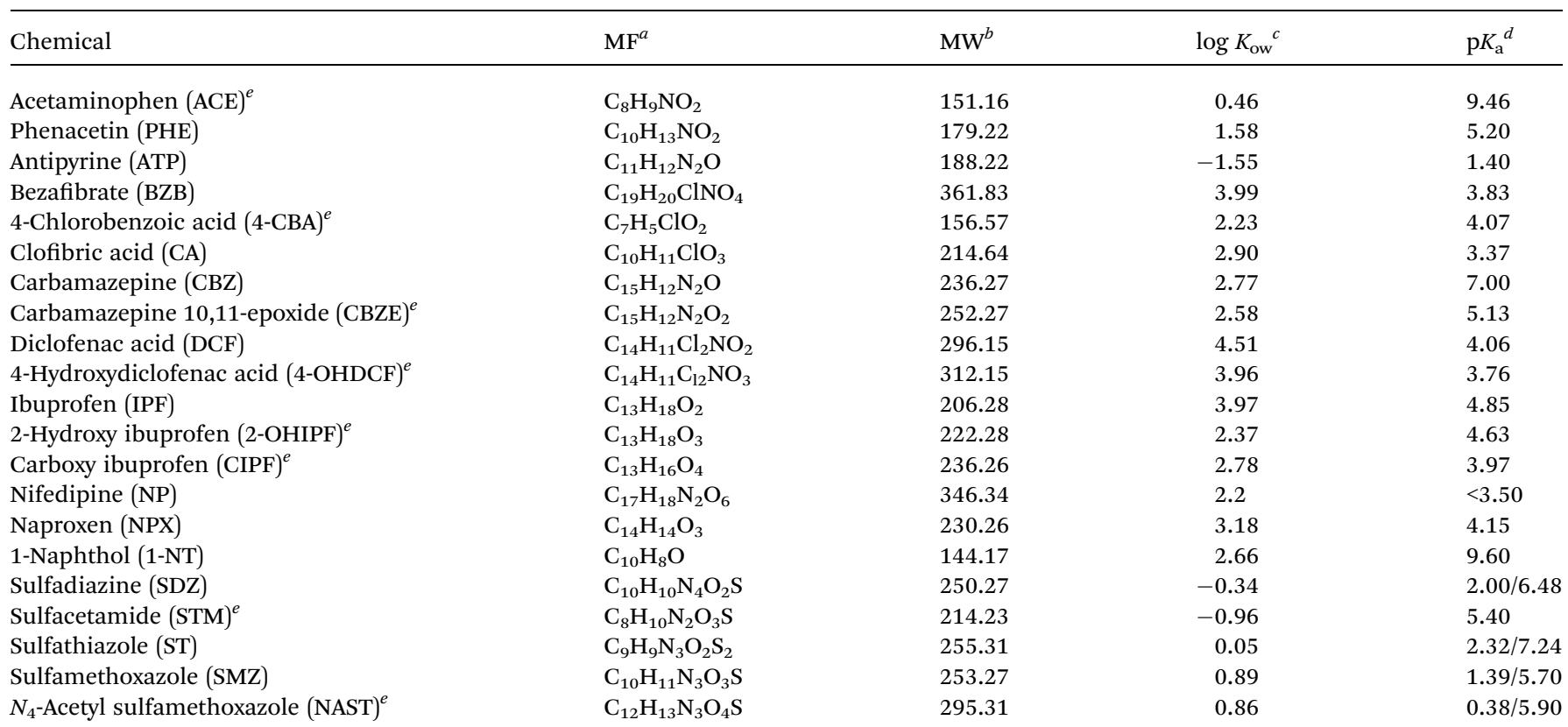

${ }^{a} \mathrm{MF}$ refer to molecular formula. ${ }^{b} \mathrm{MW}$ refer to molecular weight $\left(\mathrm{g} \mathrm{mol}^{-1}\right) .{ }^{c} K_{\mathrm{ow}}$ refer to octanol-water partition coefficient. ${ }^{d} \mathrm{p} K_{\mathrm{a}}$ refer to acid dissociation constant. ${ }^{e}$ Substances are TPs. 
the STP effluent itself (S2); $100 \mathrm{~m}$ upstream of the convergence of sewage outflow and the QH River ( $\mathrm{S} 1$, sewage flows into the $\mathrm{QH}$ River at $250 \mathrm{~m}$ ); and three points at distances of $200 \mathrm{~m}$ (S3), $1200 \mathrm{~m}$ (S4), and $3500 \mathrm{~m}$ (S5) from convergence. Water samples from the YL River were collected at four sampling sites: $100 \mathrm{~m}$ downstream of the STP outfall (E2), $200 \mathrm{~m}$ upstream of the outfall (E1), and at two sites $2000 \mathrm{~m}$ and $3000 \mathrm{~m}$ from E2. A detailed map of the sampling sites is given in Fig. 1. The water quality parameters of the four studied rivers were considerably different (Table 2).

\section{Sampling methods}

Active sampling. For a week, water samples were collected four times at all sites along each river. For each river, samples were obtained at a distance of approximately $30 \mathrm{~cm}$ below the water surface every other day using a $1.5 \mathrm{~L}$ stainless-steel water collector. A $1.0 \mathrm{~L}$ water sample was grabbed at each site, and each sample was transferred to a brown polypropylene bottle that had been previously washed with ultrapure water. All collected water samples were kept at $0{ }^{\circ} \mathrm{C}$ until the next procedure (within $24 \mathrm{~h}$ ).

Passive sampling. For each site along the QH River and YL River, three POCISs covered with stainless-steel mesh $(0.5 \mathrm{~mm})$ were deployed during the first active sampling. The POCIS was placed approximately $1.0 \mathrm{~m}$ below the water surface and withdrawn a week later. Foreign substances attached to the surface of the POCIS were rinsed off with ultrapure water, and the device was then wrapped with aluminum foil and finally stored at $0{ }^{\circ} \mathrm{C}$ until extraction (within $24 \mathrm{~h}$ ).

\section{Extraction methods}

Extraction of water samples. Procedures for extraction of water were described in detail previously. ${ }^{\mathbf{1 3 , 2 6}}$ Samples were extracted by solid-phase extraction (SPE). A $1.0 \mathrm{~L}$ water sample spiked with $100 \mu \mathrm{L}$ of internal standard solution $\left(1 \mathrm{mg} \mathrm{L}^{-1}\right.$ working solution in methanol) was extracted at each site. In the pretreatment process, water samples were filtered through a $0.45 \mu \mathrm{m}$ glass fiber filter to remove particulate matter. Oasis HLB cartridges (500 mg, $6 \mathrm{cc}$ ) were activated with $5 \mathrm{~mL}$ of methanol and then $5 \mathrm{~mL}$ of ultrapure water before loading. After SPE, the cartridges were washed with ultrapure water $(2 \times$ $5 \mathrm{~mL}$ ), dried for half an hour under vacuum to completely remove moisture from the cartridges, and finally eluted with methanol $(2 \times 5 \mathrm{~mL})$. The eluate collected was concentrated and evaporated to near dryness under a continuous nitrogen stream and reconstituted with $1 \mathrm{~mL}$ of $50: 50 \mathrm{methanol} /$ water $(\mathrm{v} / \mathrm{v})$ solution. The new eluent was filtered into a brown $1.5 \mathrm{~mL}$ chromatography bottle using a $0.22 \mu \mathrm{m}$ hydrophilic polytetrafluoroethylene syringe filter prior to analysis.

Extraction with POCIS. The extraction procedure of POCIS was based on previous report. ${ }^{13,27}$ After deployment, each POCIS was disassembled. The sorbent filler was then transferred with water from the sampler into an empty $6 \mathrm{~mL}$ SPE cartridge containing a polyethylene frit and spiked with $100 \mu \mathrm{L}$ of internal standards. Another polyethylene frit was loaded. Ultrapure water $(2 \times 5 \mathrm{~mL})$ was added to the cartridge to eliminate interference, which was then dried for half an hour under vacuum before elution. Subsequent procedures were identical to the extraction of water samples.

\section{Analytical methods}

Analysis of all target compounds was performed on an ultraperformance liquid chromatography system (Waters Acquity UPLC, USA) coupled to a triple quadrupole mass spectrometer (Waters AcquityXevo TQ) with electrospray ionization (ESI). An ultraperformance liquid chromatograph equipped with

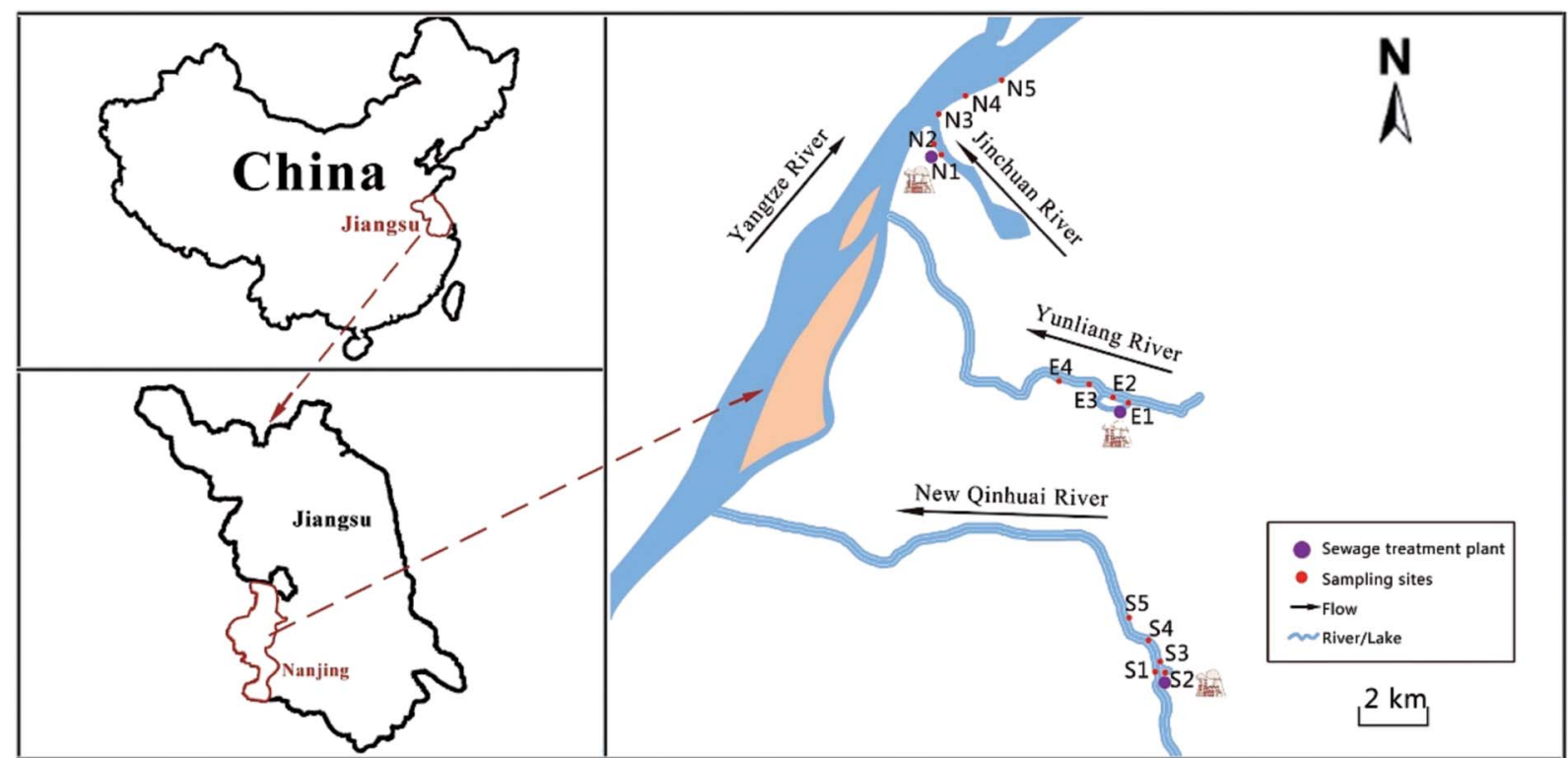

Fig. 1 Sampling locations in the studied rivers (JC, YZ, QH and YL river) marking the STPs. Sampling sites are digitally marked along the river flow direction. 
Table 2 Related parameters of four rivers

\begin{tabular}{|c|c|c|c|c|}
\hline River name & Jinchuan (JC) & Yangtze (YZ) & Qinhuai (QH) & Yunliang (YL) \\
\hline STP & North city (NC) & North city (NC) & Science park (SP) & East city (EC) \\
\hline Mean velocity ${ }^{a}\left(\mathrm{~m} \mathrm{~s}^{-1}\right)$ & 0.244 & 1.205 & 0.25 & 0.21 \\
\hline Temperature $^{b}\left({ }^{\circ} \mathrm{C}\right)$ & $15.8 \pm 0.6$ & $16 \pm 1.0$ & $11.7 \pm 0.7$ & $16.8 \pm 0.3$ \\
\hline Dissolved oxygen $^{b}\left(\mathrm{mg} \mathrm{L}^{-1}\right)$ & $7.16 \pm 0.3$ & $8.77 \pm 0.4$ & $8.73 \pm 0.4$ & $5.17 \pm 0.2$ \\
\hline Electrical conductivity $\left(\mu \mathrm{S} \mathrm{cm} \mathrm{cm}^{-1}\right)$ & $590 \pm 19$ & $600 \pm 40$ & $404 \pm 28$ & $496 \pm 55$ \\
\hline Total suspended solids ${ }^{b}\left(\mathrm{mg} \mathrm{L}^{-1}\right)$ & $13.6 \pm 3.5$ & $214 \pm 53$ & $41.2 \pm 10$ & $19.1 \pm 2.2$ \\
\hline
\end{tabular}

a Waters BEH C18 column $(2.1 \times 100 \mathrm{~mm}, 1.7 \mu \mathrm{m}$ particle diameter) was used for chromatographic separation, and the column temperature was maintained at $40{ }^{\circ} \mathrm{C}$. The target compounds were analyzed by gradient elution at a flow rate of $0.4 \mathrm{~mL} \mathrm{~min}^{-1}$ and injection volume of $5 \mu \mathrm{L}$. A $98: 2(\mathrm{v} / \mathrm{v})$ water/ methanol solution (phase A) and methanol solution (phase B), both containing $0.05 \%$ formic acid, were used as mobile phases for positive-ion mode; mobile phases of $98: 2(\mathrm{v} / \mathrm{v})$ water/methanol solution with $5 \mathrm{mM}$ ammonium acetate (phase A) and acetonitrile (phase B) were used for negative ion mode. Details of the gradient for the mobile phase and the acquisition parameters for mass spectrometry are given in Table S1 and S2 (ESI $\dagger$ ).

\section{Quality assurance and quality control}

Recovery experiments of all of the target compounds in top water and river water were performed by spiking samples to different levels. Recoveries of target compounds in top water (with concentration of 10 and $100 \mathrm{ng} \mathrm{L}^{-1}$ ) were at a satisfactory range of between 62.4 (NP) and 135.36\% (4-OHDCF) (Table S3†) and recoveries in river water (with concentration of $100 \mathrm{ng} \mathrm{L}^{-1}$ ) ranged from $48 \%$ for 4 -CBA to $126.5 \%$ for IPF (Fig. S1 $\dagger$ ). Recoveries were computed by comparing the concentrations measured in the sample after the extraction process with the initial spiked level. The concentrations measured in the sample were determined by using internal sample calibration. Blank control experiments containing only the internal standard were analyzed; here, no target substances were detected. For passive sampling during POCIS deployment in the field, unexposed blank POCISs $(n=3)$ wrapped with aluminum foil were placed in the refrigerator, and analysis was performed when the field exposure experiment was finished. Moreover, the blank filler (HLB, $200 \mathrm{mg}$ ) was analyzed in order to determine background concentration of each target compound. In addition, the solvent blank was analyzed. None of the target compound was detected in the blanks. For active sampling, analogous blank experiments were done and no target substance was detected.

Limits of detection and limits of quantification (LOQ), defined as the lowest concentrations corresponding to signal-tonoise ratios of 3 and 10, respectively (Table S3†), were determined. The standard deviations from the recovery experiments were $<14 \%$.

\section{Calculations of attenuation rate}

The attenuation rates of pharmaceutical and their TPs along the river indicate their concentration changes relative to a compound, which can remain for long periods in the aquatic environment. ${ }^{15}$ This means that concentrations at the downstream site compared to the upstream site are expected to be equal for persistent compounds or lower for substances affected by loss processes. We used CBZ, a compound that is widely detected in sewage and has a long half-life in aquatic systems. ${ }^{28,29}$ The RA value of a substance was computed by dividing its concentration at site 2 by the concentration at site 1 . The ratio was then normalized by the concentration ratio of CBZ at both sites and finally multiplied by $100 \%$. A positive value indicates that the pharmaceutical is attenuated along the river, whereas a negative value indicates an increase in concentration. A value equal to zero indicates that the substance is persistent or that its attenuation rate is approximately equal to its rate of production. The RA (\%) was calculated as follows: ${ }^{13,15,30}$

$$
\mathrm{RA}=\left(1-\frac{\frac{C_{\mathrm{S}, \text { site } 2}}{C_{\mathrm{S}, \text { site } 1}}}{\frac{C_{\mathrm{P}, \text { site } 2}}{C_{\mathrm{P}, \text { site } 1}}}\right) \times 100 \%
$$

where $C_{\mathrm{S} \text {, site } 1}$ and $C_{\mathrm{S} \text {,site } 2}$ are the concentrations $\left(\mathrm{ng} \mathrm{L}^{-1}\right)$ of the substance at sites 1 and 2 , respectively; $C_{\mathrm{P} \text {,site } 1}$ and $C_{\mathrm{P}, \text { site } 2}$ are the concentrations ( $\mathrm{ng} \mathrm{L}^{-1}$ ) of the persistent substance $\mathrm{CBZ}$ at sites 1 and 2, respectively. In this study, sites 1 and 2 respectively correspond to N3 and N5 for YZ River, E2 and E4 for YL River, and S3 and S5 for QH River. There was not major tributaries or additional sources of target compounds between these two points. For the calculations, pharmaceuticals with concentrations below LOQ at site 2 were assumed to be the LOQ concentration. Thus, the attenuation rate was likely to be an underestimate.

For the attenuation of substances on the POCIS, because of the concentration from POCIS is calculated by the formula, and require calibration of the POCIS. Moreover, calibration results depend on the hydrological conditions of the river. Therefore, we computed the RA of the compound from the POCIS by considering its enrichment in the POCIS. This method does not need calibration of the POCIS, and it assumes that the uptake kinetics of analytes at the two sites is the same. 


\section{Results and discussion}

\section{Occurrence of pharmaceuticals and their TPs in the four rivers}

Among the target compounds, CIPF and STM were not detected in all sampling sites; hence, they are not discussed here. Twelve target compounds were detected at all sampling sites in the four rivers. NP was the only one that was detected in sampling site $\mathrm{N} 2$, which was the outlet of NC STP. This result implies that NP is derived only from STP. CA, ST, NPX, and DCF were not detected in the QH River, but DCF and NPX were detected at the STP outlet. Concentrations of all analytes that were detected at sites 1 and 2 are shown in Fig. 2, and concentrations of the detected substances at all sites are listed in Table S4 (ESI $\dagger$ ). In general, the JC River and YZ River, which were affected by NC STP, had a high concentration of target compounds. After all, NC STP has a dense population around it, a majority of pharmaceuticals caused by people were transported to the STP, so that the STP discharges a large amount of wastewater containing high concentrations of contaminants; concentrations of the detected compounds at $\mathrm{N} 2$ ranged from $0.68 \mathrm{ng} \mathrm{\textrm {L } ^ { - 1 }}$ (CA) to $544.0 \mathrm{ng} \mathrm{\textrm {L } ^ { - 1 }}$ (CBZE). Moreover, the concentrations of DCF, CBZ, and IPF were essentially the same as those in a study by Liu et al. ${ }^{26}$ who analyzed concentrations of these substances downstream of the NC STP outlet. Upstream of the NC STP outlet, most of the compounds were detected at high concentrations, with some even higher than the outlet concentration (such as ACE, IPF, SMZ, NAST). Besides the effluent from STP, the site located upstream is also a source of these substances. For the YZ River, the analyte concentrations at site 1 (N3) ranged from $1.1 \mathrm{ng} \mathrm{L}^{-1}$ (CA) to $448.0 \mathrm{ng} \mathrm{\textrm {L } ^ { - 1 }}$ (CBZE). NP and 1-NT were not detected there, but they were detected in the JC River. The concentration of the majority of these substances in the outlet of the QH River was substantially lower than that at the NC STP outlet. The highest concentration detected was $124.0 \mathrm{ng} \mathrm{\textrm {L } ^ { - 1 }}$ for ACE; 4-OHDCF reached the lowest concentration, $1.4 \mathrm{ng} \mathrm{\textrm {L } ^ { - 1 }}$. ACE is metabolite of PHE, which is widely used as an analgesic and antipyretic around the world. PHE has a high concentration

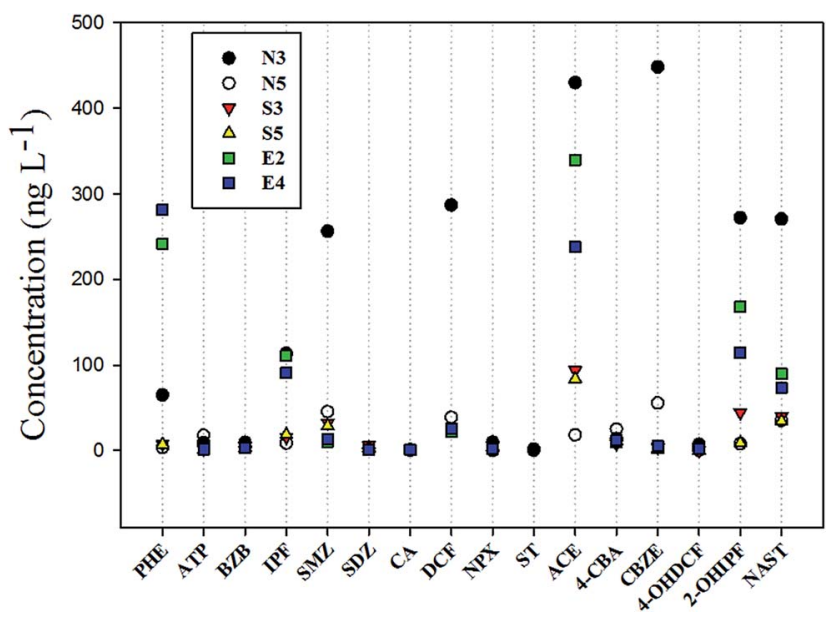

Fig. 2 Concentration from active sampling of 16 compounds detected at sampling sites 1 and 2. ACE, 4-CBA, CBZE, 4-OHDCF, 2-OHIPF and NAST are TPs. in rivers receiving treated wastewater; ${ }^{31,32}$ it was widely detected in wastewater, surface water, groundwater, and even drinking water. $^{32,33}$ The concentration distribution of other sampling points in QH River is shown in Table S4. $\dagger$ The concentrations of these compounds at E2 along the YL River ranged from $0.43 \mathrm{ng} \mathrm{L}{ }^{-1}$ (SDZ) to $244.0 \mathrm{ng} \mathrm{L}^{-1}$ (PHE). The concentrations of most of the compounds at most sampling points were higher than those in the QH River (Table S4†).

\section{Attenuation of pharmaceuticals and their TPs}

The attenuation data were obtained from points 1 and 2. The change in concentration between these two points may be attributed to dilution due to inflow of surface water or to the elimination or formation of these compounds by various factors downstream of the river. The calculation of attenuation rate relative to that of $\mathrm{CBZ}$, a persistent compound, can be done to explain the dilution; RA $>0$ indicates attenuation. The succeeding discussion is based on the results of active sampling at the YZ, QH, and YL Rivers.

Attenuation of parent compounds. Eleven parent pharmaceuticals were attenuated in at least one river (Fig. 3). In the YZ River, RA values ranged from $-164 \%$ (ATP) to $94.1 \%$ (NPX), and the attenuation rates of PHE, IPF, SMZ, DCF, NPX, and ST were higher than those in the other two rivers. YZ River provides environment conditions for attenuation that are more favorable than those in the other two rivers. First, the total suspended solids and the flow rate of the YZ River are much higher than those of the QH River and YL River (Table 2). Absorption of the compounds by suspended solids can cause the detected concentrations to be lower than the actual. The high flow rate hastens the diffusion of these compounds and indirectly affects the attenuation. Second, YZ River has less shading along the studied stretch, thus it is more illuminated compared with the other two rivers. And it also provided more favorable conditions for light penetration into the water column, the condition that

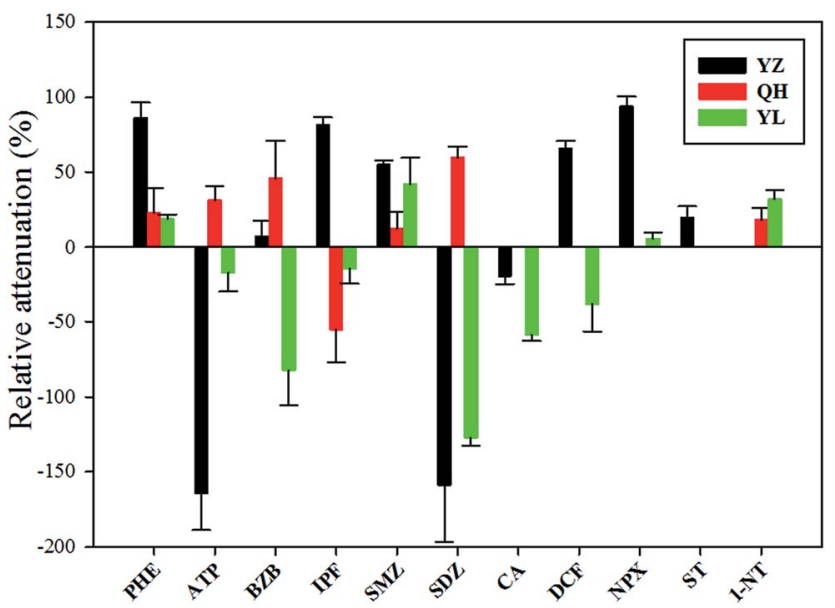

Fig. 3 Relative attenuation (RA, \%) of these parent pharmaceuticals detected from site 1 to site 2 in the rivers based on samples collected with active sampling. It is shown that the eleven of 13 parent pharmaceuticals that were detected in at least one river. No data indicates that the substance was not detected at two points. 
directly or indirectly aids the photodegradation of these substances. Photodegradation was not the dominant attenuation mechanism for most of the substances in forested rivers such as QH River and YL River, which were mostly shaded by trees and buildings. Although photolysis may be an important attenuation mechanism, biotransformation may be as important as or more significant than is photodegradation because of light attenuation over depths or because of absorption of suspended solids. ${ }^{\mathbf{3 4 3 5}}$ This is particularly the case with the YZ River; biotransformation is a significant removal process in this large river. ${ }^{35}$ But for more photolabile compounds, photodegradation may still be the dominant removal mechanism in deeper rivers. The attenuation of NPX was likely underestimated because its concentration at N3 was less than LOQ; for the calculations, its concentration was assumed to be the LOQ. In a sunlit river, photodegradation is the dominant attenuation process for NPX, resulting in a half-life of less than 1 h. ${ }^{34}$ Negative RA values for active pharmaceutical ingredients are only possible, if a specific source for the target is active along the flow path. This seems to happen for ATP and SDZ in the Yangtze (Fig. 3); thus, there may be a wide variety of pharmaceuticals in the YZ River or there may be a small tributary that is hard to find near this. Among the parent pharmaceutical compounds detected in the $\mathrm{QH}$ River, IPF was the only one without attenuation, and concentrations at five sampling points (S1-S5) were not greatly different. PHE, ATP, BZB, SMZ, SDZ, and 1-NT were attenuated; their attenuation rates ranged from $12.2 \%$ (SMZ) to $59.6 \%$ for (SDZ) in the QH River. The partial loss of pharmaceuticals may be attributed to sorption by sediments; it may be a significant mechanism resulting in the comparatively high attenuation rates for a number of substances. ${ }^{36-38}$ We can conclude that the sediment provided favorable conditions for the attenuation of these substances in the QH River. But partition of hydrophilic compounds to sediments is anyhow rather low as is biotransformation in river water. Furthermore, river boundary conditions such as shallow depth, low turbidity, and sandy sediments aid phototransformation and biotransformation of these substances. ${ }^{15}$ In the YL River, only PHE, SMZ, NPX, and 1-NT were attenuated. RA values of ATP, BZB, IPF, SDZ, CA, and DCF were $<0$, indicating that the concentrations of these substances increased from E2 to E4. The main cause of the increase in concentrations was the discharge of wastewater from STP; furthermore, there might be other sources of these substances. Many people live on both sides of the YL River, and they put most of their garbage into the river. Moreover, part of the domestic sewage containing these substances may flow into the river. Therefore, the YL River may be more contaminated than were the other two rivers.

The attenuation of pharmaceuticals in the three rivers was analyzed. Most of the pharmaceuticals except for ATP, SDZ, and CA in the YZ River were more susceptible to attenuation than they were in the other rivers (Fig. 3). Attenuation conditions for these substances might have been favorable in the YZ River, that is, favorable light and microbial conditions, fast flow velocity, and so forth. River flow rate has a significant effect on the attenuation, with higher velocity of water resulting in a larger RA. ${ }^{36}$ On the other hand, a large amount of sewage discharged from the NC STP into the YZ River (previous study shows that BOD, COD and TOC were $1.73 \mathrm{mg} \mathrm{L}^{-1}, 24.4 \mathrm{mg} \mathrm{L}^{-1}$ and $15.05 \mathrm{~g} \mathrm{~kg}^{-1}$, respectively ${ }^{39}$ ) contributes to a multitude of microorganisms and bacteria in the water, which can form biofilms at the water-sediment interface. Both processes absorb and transform organic micropollutants. ${ }^{\mathbf{4 0 4 1}}$ Moreover, the total organic carbon present in the sediments may affect the attenuation of these substances; low total organic carbon content leads to high attenuation. ${ }^{\mathbf{1 3 , 3 6}}$ DCF, NPX, and SMZ were attenuated in at least one river. Studies have shown that DCF and NPX tend to undergo photodegradation, having a short half-life in sunlit river; SMZ tends to undergo biodegradation..$^{\mathbf{3 4 4 2 , 4 3}}$ The photolysis of SMZ has been shown to be strongly pH dependent and higher photolysis at lower $\mathrm{pH},{ }^{\mathbf{4 4}}$ and studied rivers have relatively high $\mathrm{pH}$ values (Table 2 ). Consequently, it conclude that photodegradation contributes little to the total removal of SMZ in rivers. However, photodegradation processes are complex, we have to further explore the process to provide more information on the removal by photolysis.

The attenuation pattern of the substances in the three rivers were varied and complex. In order to understand the relationship between the physical and chemical properties of these substances and their attenuation rates, relevant analysis was conducted. The results show that no correlation between them $(p>0.05$, Table S5 $\dagger)$; hence, the physical and chemical properties of these substances do not reflect the attenuation. In the YZ River, the flow rate, photodegradation, biotransformation, and total suspended solids may lead to attenuation. However, sorption to suspended solids cannot be an attenuation process for these substances in the QH River and YL River because the particle load of the water samples was low. Therefore, biotransformation and adsorption of sediments and photolysis can result in attenuation in the $\mathrm{QH}$ and YL River. However, comparison between different rivers is complicated due to the different hydraulics and sediments.

Attenuation of TPs. All TPs detected except 4-CBA were attenuated in the three rivers (Fig. 4). In theory, it can observe the formation of TPs due to the transformation of parent pharmaceutical compounds, but we detected only 4-CBA. The attenuation rates of the remaining TPs were $>0$ in the three rivers, and the TPs concentrations at site 1 were relatively high. The attenuation rates of 4 -CBA were $<0$ in the three rivers, which indicates an increase in concentration. The trend of attenuation is the same as that in previous studies. ${ }^{13}$ The increase in 4-CBA concentration suggests the transformation of its parent molecule, BZB, but the 4-CBA concentration at sites 1 and 2 is much higher than that of $\mathrm{BZB}$; therefore, the accumulation of 4-CBA is not entirely due to BZB. Part of the 4-CBA was may be derived from the transformation of other substances such as pesticides and herbicides ${ }^{\mathbf{4 5 4 6}}$ or from other sources at sampling sites. CBZE, 4-OHDCF, 2-OHIPF, and NAST were attenuated at different levels in the three rivers; the maximum attenuation rates of these TPs were appear in the YZ River, which provided favorable conditions for attenuation, followed by those observed in the $\mathrm{QH}$ River and YL River. The minimum attenuation rate in the $\mathrm{QH}$ River was that for ACE, showing that ACE in this river environment did not tend to attenuate or there were 


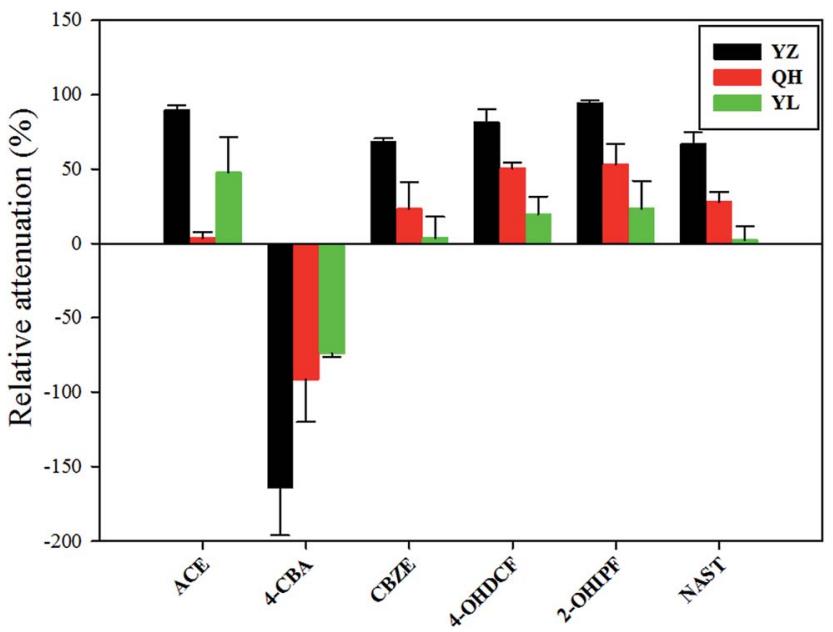

Fig. 4 Relative attenuation (RA, \%) of transformation products (TPs) detected from site 1 to site 2 in the three rivers based on active sampling of samples.

other sources of ACE. This difference may be due to the much higher concentrations of ACE at sites 1 and 2 compared with those of its parent compound (Table S4 †).

Although several parent compounds were attenuated downstream of the rivers, the corresponding TPs (except 4CBA) did not increase in concentration. This result may be explained as follows: during transformation of the parent compounds, the resulting TPs were attenuated because of their short half-life. Because the sampling period between sites 1 and 2 (correspond to N3 and N5 for YZ, E2 and E4 for YL, S3 and S5 for QH) was short $(<24 \mathrm{~h})$, transformation of all parent compounds during this period is unlikely. Thus, we can conclude that the vast majority of these TPs were due to the discharge of STP.

\section{Comparison of attenuation results from active and passive sampling}

The purpose of comparing the attenuation results from active and passive sampling was to determine whether passive sampling can be applied to compute the attenuation of these substances and perhaps organic micropollutants in rivers. Attenuation rates of pharmaceuticals and their TPs from active and passive sampling in the QH River and YL River are shown in Fig. 5 (details are given in Table $56 \dagger$ ). The $83 \%$ confidence intervals of the five pharmaceuticals and five their TPs overlap with the $1: 1$ line, indicating good agreement between attenuation rates calculated from the two sampling approaches. Results for ACE and 4-CBA from active and passive sampling in the YL River were also markedly different. The computed rates for ACE and 4-CBA from passive sampling were $89.1 \%$ and $30.5 \%$, respectively, and those from active sampling were $47.6 \%$ and $-73.4 \%$, respectively. The former value was greater than the latter. The 4-CBA concentration increased during active sampling, and it decreased during passive sampling. The river flow rate has an impact on the rates of POCIS uptake, ${ }^{47,48}$ but an additional performance reference compound (PRC) needs to be

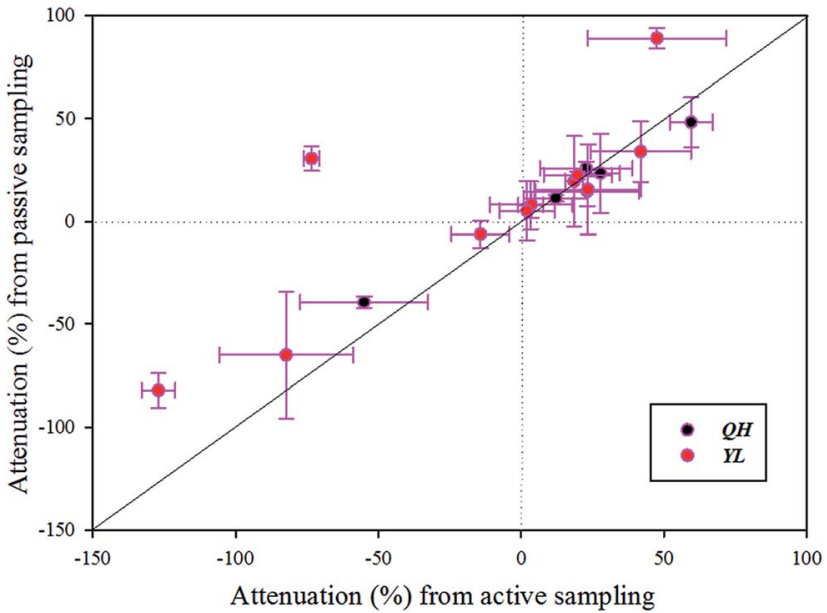

Fig. 5 Relative attenuation (RA, \%) in the $\mathrm{QH}$ and $\mathrm{YL}$ river derived from active and passive sampling for part of analytes. The solid $1: 1$ line indicates that the results from active and passive sampling are identical.

used to correct for flow rate effects. Another explanation could be additional inputs of these two compounds between E2 and E4 during periods of time that were not captured by grab sampling but were continuously accumulated by deployed POCIS. The attenuation rates for BZB, SDZ, and IPF were negative. Although the values were slightly different, they showed the same attenuation trend as that obtained with active sampling. Active sampling was thus likely to be instantaneous sampling, and passive sampling involved continuous accumulation of contaminants. Overall, passive sampling with an appropriate PRC may be a reliable method for monitoring attenuation of most of the compounds.

\section{Conclusions}

In this study, the occurrence and attenuation of pharmaceuticals and their TPs in rivers receiving treated wastewater were investigated. The results show that 19 of 21 compounds were detected at in least one sampling point in each river. The maximum concentration of the outlet of the STP was $544.0 \mathrm{ng}$ $\mathrm{L}^{-1}$ (CBZE), and the minimum value was $0.43 \mathrm{ng} \mathrm{L}^{-1}$ (SDZ). The majority of the pharmaceuticals and their TPs were attenuated in the rivers except in the YL River. Activities of the residents along the river might have affected the river, resulting in negative attenuation for some of the compounds. Photodegradation, sorption to sediments, and biotransformation may be the major removal mechanisms for many of the compounds. Results from active and passive sampling suggest that passive sampling may be a reliable alternative to spot sampling for determining the attenuation rates of many compounds. Passive sampling can also be used for long-term monitoring, which is too resource demanding by spot sampling. However, its potential for measuring the attenuation rates of more organic micropollutants needs in-depth exploration. 


\section{Conflicts of interest}

There are no conflicts to declare.

\section{Acknowledgements}

The presented study was supported by the National Natural Science Foundation of China (No. 51479067), the Fundamental Research Funds for the Central Universities (No. 2017B13514) and the Project Founded by the Priority Academic Program Development of Jiangsu Higher Education Institutions.

\section{References}

1 J. Wang and L. Chu, Radiat. Phys. Chem., 2016, 125, 56-64. 2 J. L. Liu and M. H. Wong, Environ. Int., 2013, 59, 208-224.

3 P. J. Ferguson, M. J. Bernot, J. C. Doll and T. E. Lauer, Sci. Total Environ., 2013, 458-460, 187-196.

4 D. Alvarez-Munoz, B. Huerta, M. Fernandez-Tejedor, S. Rodriguez-Mozaz and D. Barcelo, Talanta, 2015, 136, 174-182.

5 J. Rivera-Utrilla, M. Sanchez-Polo, M. A. Ferro-Garcia, G. Prados-Joya and R. Ocampo-Perez, Chemosphere, 2013, 93, 1268-1287.

6 H. Auvinen, I. Havran, L. Hubau, L. Vanseveren, W. Gebhardt, V. Linnemann, D. Van Oirschot, G. Du Laing and D. P. L. Rousseau, Ecol. Eng., 2017, 100, 157-164.

7 E. Vulliet and C. Cren-Olive, Environ. Pollut., 2011, 159, 29292934.

8 J. Fick, H. Soederstrom, R. H. Lindberg, C. Phan, M. Tysklind and D. G. J. Larsson, Environ. Toxicol. Chem., 2009, 28, 25222527.

9 E. Godfrey, W. W. Woessner and M. J. Benotti, Ground Water, 2007, 45, 263-271.

10 U. Hass, U. Duennbier and G. Massmann, Water Res., 2012, 46, 6013-6022.

11 M. D. Celiz, J. Tso and D. S. Aga, Environ. Toxicol. Chem., 2009, 28, 2473-2484.

12 L. Schlüter-Vorberg, C. Prasse, T. A. Ternes, H. Mückter and A. Coors, Environ. Sci. Technol. Lett., 2015, 2, 342-346.

13 Z. Li, A. Sobek and M. Radke, Environ. Sci. Technol., 2016, 50, 5614-5621.

14 M. Rodríguez-Delgado, C. Orona-Navar, R. García-Morales, C. Hernandez-Luna, R. Parra, J. Mahlknecht and N. Ornelas-Soto, Int. Biodeterior. Biodegrad., 2016, 108, 3441.

15 U. Kunkel and M. Radke, Water Res., 2012, 46, 5551-5565.

16 V. Acuna, D. von Schiller, M. J. Garcia-Galan, S. RodriguezMozaz, L. Corominas, M. Petrovic, M. Poch, D. Barcelo and S. Sabater, Sci. Total Environ., 2015, 503-504, 133-141.

17 A. M. Greenhagen, M. E. Lenczewski and M. Carroll, Chemosphere, 2014, 115, 13-19.

18 E. Brezina, C. Prasse, J. Meyer, H. Muckter and T. A. Ternes, Environ. Pollut., 2017, 225, 261-269.

19 O. Koba, O. Golovko, R. Kodesova, A. Klement and R. Grabic, Environ. Pollut., 2016, 218, 574-585.
20 I. Aymerich, V. Acuna, D. Barcelo, M. J. Garcia, M. Petrovic, M. Poch, S. Rodriguez-Mozaz, I. Rodriguez-Roda, S. Sabater, D. von Schiller and L. Corominas, Water Res., 2016, 100, 126-136.

21 B. F. Da Silva, A. Jelic, R. Lopez-Serna, A. A. Mozeto, M. Petrovic and D. Barcelo, Chemosphere, 2011, 85, 13311339.

22 J. H. Writer, R. C. Antweiler, I. Ferrer, J. N. Ryan and E. M. Thurman, Environ. Sci. Technol., 2013, 47, 9781-9790.

23 A. Vallejo, A. Prieto, M. Moeder, A. Usobiaga, O. Zuloaga and N. Etxebarria, Water Res., 2013, 47, 2851-2862.

24 I. Ibrahim, A. Togola and C. Gonzalez, Talanta, 2013, 116, 495-500.

25 J. A. Baz-lomba, C. Harman, M. Reid and K. V. Thomas, Water Res., 2017, 121, 221-230.

26 J. C. Liu, G. H. Lu, Z. X. Xie, Z. H. Zhang, S. Li and Z. H. Yan, Sci. Total Environ., 2015, 511, 54-62.

27 S. L. Kaserzon, K. Kennedy, D. W. Hawker, J. Thompson, S. Carter, A. C. Roach, K. Booij and J. F. Mueller, Environ. Sci. Technol., 2012, 46, 4985-4993.

28 V. Calisto, M. R. M. Domingues, G. L. Erny and V. I. Esteves, Water Res., 2011, 45, 1095-1104.

29 H. Zou, M. Radke, A. Kierkegaard, M. MacLeod and M. S. McLachlan, Environ. Sci. Technol., 2015, 49, 1646-1653.

30 Z. Li, S. L. Kaserzon, M. M. Plassmann, A. Sobek, M. J. Gomez Ramos and M. Radke, Environ. Sci.: Processes Impacts, 2017, 19, 488-498.

31 X. Zhang, F. Wu, X. W. Wu, P. Chen and N. Deng, J. Hazard. Mater., 2008, 157, 300-307.

32 P. H. Roberts and K. V. Thomas, Sci. Total Environ., 2006, 356, 143-153.

33 C. Wang, H. Shi, C. D. Adams, S. Gamagedara, I. Stayton, T. Timmons and Y. Ma, Water Res., 2011, 45, 1818-1828.

34 A. Y. C. Lin and M. Reinhard, Environ. Toxicol. Chem., 2005, 24, 1303-1309.

35 L. J. Fono, E. P. Kolodziej and D. L. Sedlak, Environ. Sci. Technol., 2006, 40, 7257-7262.

36 Y. Luo, L. Xu, M. Rysz, Y. Q. Wang, H. Zhang and P. J. J. Alvarez, Environ. Sci. Technol., 2011, 45, 1827-1833.

37 B. Li and T. Zhang, Environ. Sci. Technol., 2010, 44, 34683473.

38 A. Y. C. Lin, M. H. Plumlee and M. Reinhard, Environ. Toxicol. Chem., 2006, 25, 1458-1464.

39 W. R. Liu, J. L. Zhao, Y. S. Liu, Z. F. Chen, Y. Y. Yang, Q. Q. Zhang and G. G. Ying, Environ. Pollut., 2015, 200, 5363.

40 D. Wicke, U. Bockelmann and T. Reemtsma, Water Res., 2007, 41, 2202-2210.

41 J. H. Writer, J. N. Ryan and L. B. Barber, Environ. Sci. Technol., 2011, 45, 7275-7283.

42 S. Poirier-Larabie, P. A. Segura and C. Gagnon, Sci. Total Environ., 2016, 557-558, 257-267.

43 T. Su, H. Deng, J. P. Benskin and M. Radke, Chemosphere, 2016, 148, 518-525.

44 D. E. Moore and W. Zhou, Photochem. Photobiol., 1994, 59, 497-502. 
45 Y. Magara, T. Aizawa, N. Matumoto and F. Souna, Water Sci. Technol., 1994, 30, 119-128.

46 R. Müller and F. Lingens, Angew. Chem., Int. Ed., 1986, 25, 48 M. Di Carro, L. Bono and E. Magi, Talanta, 2014, 120, 30779-789.
47 C. M. Yang, Y. Li, D. P. Zha, G. H. Lu, Q. Sun and D. H. Wu, Chemosphere, 2017, 167, 1-9.

33. 